\title{
Three-dimensional contrast-enhanced magnetic resonance angiography for anterolateral thigh flap outlining: A retrospective case series of 68 patients
}

\author{
CHUNJING JIANG ${ }^{1 *}$, PING LIN ${ }^{2 *}$, XIAOYAN FU ${ }^{3}$, JINER SHU ${ }^{1}$, HUIMIN LI $^{4}$, \\ XIAOGANG HU ${ }^{2}$, JIANRONG HE ${ }^{1}$ and MINGXING DING ${ }^{3}$
}

Departments of ${ }^{1}$ Radiology and ${ }^{2}$ Hand-Foot Surgery, Jinhua Municipal Central Hospital, Jinhua, Zhejiang 321000;
${ }^{3}$ Department of Medical Sciences, Jinhua College of Profession and Technology, Jinhua, Zhejiang 321007;
${ }^{4}$ Department of Radiology, Xinhua Hospital, Shanghai Jiaotong University School of Medicine,
Shanghai 200092, P.R. China

Received September 19, 2014; Accepted July 14, 2015

DOI: $10.3892 /$ etm.2016.3387

\begin{abstract}
Flap transfer is increasingly used for repairing limb defects secondary to trauma or tumor, and appropriate preoperative planning plays a critical role. The present study aimed to examine the use of three-dimensional (3D) contrast-enhanced magnetic resonance angiography (CE-MRA) in evaluating the blood supply distribution and perforating branch pattern of anterolateral thigh (ALT) flaps. Bilateral donor lower limbs were scanned in 68 patients (136 limbs) using a Siemens Avanto 1.5 T magnetic resonance imaging scanner with a 3D fast low-angle shot sequence, following the thin-slab maximum intensity projection (TS-MIP) technique. The lateral femoral circumflex artery (LFCA) was visualized in all patients: 101 limbs $(101 / 136,74.3 \%)$ were type I; 20 limbs $(20 / 136$, $14.7 \%)$ were type II; 3 limbs $(3 / 136,2.2 \%)$ were type III; and 12 limbs (12/136, 8.8\%) were type IV. Tertiary branches were identified in 94 limbs $(94 / 136,69.1 \%)$. Donor flaps were outlined according to MRA TS-MIP findings in 4 patients. All flaps survived uneventfully following the transfer. In donor flap outlining, 3D CE-MRA with the TS-MIP technique allowed an accurate, direct visualization of the branching pattern and distribution profile of the LFCA supplying the ALT flap.
\end{abstract}

Correspondence to: Professor Mingxing Ding, Department of Medical Sciences, Jinhua College of Profession and Technology, 1188 Wuzhou Street, Jinhua, Zhejiang 321007, P.R. China

E-mail: mxding@hotmail.com

*Contributed equally

Key words: magnetic resonance angiography, contrast-enhanced, lateral femoral circumflex artery, flap, three-dimensional reconstruction

\section{Introduction}

Large-area cutaneous and subcutaneous tissue defects caused by surgeries for trauma or tumor are often refractory to repair and induce a high disability rate, seriously impairing the physical and sociopsychological wellbeing of patients. The repair of these defects usually requires the microsurgical transfer of skin flaps. In 1985 and 1987, Luo (1) and Chen (2) reported the use of a free anterolateral thigh flap (FALTF) with a lateral femoral circumflex artery (LFCA) pedicle in the repair of large-area soft tissue defects. This type of flap has been frequently used in head and neck reconstruction $(3,4)$. The descending branch of the LFCA is the most common blood supply of the anterolateral thigh (ALT). The musculocutaneous or intermuscular perforator arteries of the LFCA descending branch primarily nourish the ALT skin; however, these perforator arteries exhibit small orifices and high inter-individual variability, leading to challenges in the preoperative localization of these vessels, and subsequently complicating FALTF dissection and harvest (5). Accurate preoperative assessment of ALT perforating vessels therefore plays a critical role in a successful FALTF transfer, as this enables surgeons to understand the pattern, intermuscular orientation and convergence of perforating vessels.

Advances in medical imaging technologies allow the visualization of LFCA perforating vessels in a three-dimensional (3D) manner. Multiple techniques, such as digital subtraction angiography (DSA), computed tomography angiography (CTA) and magnetic resonance angiography (MRA) (6), have been used to localize perforating vessels for the purpose of flap harvest and transfer. Among these techniques, CTA is considered to be the mainstay technique for the preoperative assessment of FALTF transfer, according to the current literature. Previous studies have shown that the use of CTA in assisting flap outlining decreased the risk of complications and increased the repair success rate (6-8). Contrast-enhanced 3D MRA (3D-CE-MRA) is a highly accurate, specific imaging tool for the diagnosis of arterial disorders (9), although it has rarely been used in FALTF transfer for repairing large-area soft 
tissue defects (6). The present study aimed to retrospectively investigate the effectiveness of 3D-CE-MRA for visualizing the 3D anatomy of the LFCA and its perforating vessels in 68 patients scheduled for elective FALTF transfer.

\section{Patients and methods}

Patients. The study protocol was approved by the Institutional Review Board of Jinhua Municipal Central Hospital (Jinhua, China). Sixty-eight patients scheduled for elective FALTF transfer were consecutively referred to the imaging center for 3D-CE-MRA of the bilateral lower limbs between September 2009 and November 2010. This retrospective patient cohort comprised 53 males and 15 females, aged 27-91 years (mean, 65 years). A wide range of vascular tree imaging analyses were conducted on the lower limb extremities of 50 patients. Among these, nine cases of vascular tree imaging were only taken from the iliac artery to the knee arteries, due to calf vein pollution. This facilitated surgical evaluation. The indications for 3D-CE-MRA were as follows: i) Having no known lower limb vascular disease; and ii) being scheduled for FALTF transfer of which 10 patients later underwent FALTF surgery. FALTF transfer is a flap surgical procedure used to repair large areas of skin and tissue defects. The contraindications were i) lower limb arteriosclerosis obliterans; ii) renal insufficiency; and iii) ineligibility for MR imaging (MRI). Among these patients, 50 underwent large-scale angiography of the entire bilateral lower limbs, 9 patients underwent angiography from the common iliac artery to the superior genicular artery only, due to calf venous contamination, and 9 patients underwent angiography from the external iliac artery to the superior genicular artery only. All patients gave informed written consent prior to participation in this study.

3D-CE-MRA. A Magnetom Avanto 1.5 T MRI system (Siemens Medical Solutions, Erlangen, Germany), equipped with a total image matrix peripheral vascular MRI coil and an integrated flexible coil, was used for 3D-CE-MRA at a switching rate of $200 \mathrm{mT} / \mathrm{msec}$ and a gradient field of $45 \mathrm{mT} / \mathrm{m}$. The bilateral lower limbs were scanned from the common iliac artery to the superior genicular artery or from the renal artery to the dorsalis pedis artery, using a fast low-angle shot gradient-echo sequence. An angiocatheter (Linhua Medical Devices Co. Ltd., Suzhou, China) was inserted into the antecubital vein, and a $30-\mathrm{ml}$ gadolinium-diethylene triamine penta-acetic acid bolus (0.2-0.3 mmol $/ \mathrm{kg}$; Beilu Pharmaceutical Co. Ltd., Beijing, China) was injected at a rate of 2.5-4.0 ml/sec using a high-pressure syringe pump (Ulrich Medical, Ulm, Germany), followed by rinsing with normal saline. LFCA MRA was started at a single-sequence time of $16 \mathrm{sec}$, immediately following the peak time of the common or internal/external iliac artery MRA on real-time Siemens Care Bolus sequence (Siemens Medical Solutions) tracking, at a repetition time (TR) of $32.56 \mathrm{msec}$, echo time (TE) of $1.19 \mathrm{msec}$ and a slice thickness of $40 \mathrm{~mm}$. Angiograms were captured in three segments using an auto-shifting bed technique to automatically reconstruct the entire angiogram of the vascular tree of the bilateral lower limb. The post-dosing FLASH 3D-core post-angiogram was subtracted from the pre-dosing FLASH $3 \mathrm{D}$-core pre-angiogram to reconstruct the LFCA angiogram using the following parameters: TR, $2.94 \mathrm{msec}$; TE, $1 \mathrm{msec}$; flip angle, $25^{\circ}$; slices per slab, 88 ; slice thickness, $1.40 \mathrm{~mm}$; distraction factor, 20\%; and fat suppression, none.

Image processing and data analysis. All captured image data were transmitted to a Syngo B17.0 3D image analysis workstation (Siemens Medical Solutions), and the arteries of the bilateral thighs were reconstructed in 3D using the maximum intensity projection (MIP), incorporating thin-slab MIP (TS-MIP), following the digital subtraction. TS-MIP is a technique used to determine the thickness of an MIP layer. Non-subtractive reconstruction was concomitantly used to visualize muscular and subcutaneous soft tissues.

Two board-certified radiovascular surgeons and two board-certified plastic surgeons were assigned to review the LFCA 3D-CE-MRA images in a double-blind manner to evaluate the origin pattern of the LFCA and the distribution, orientation and convergence of perforating vessels, as well as the length of the vascular pedicle. Anatomical patterns of the LFCA were classified into four types, as previously described $(10,11)$ : Type Ia, the LFCA originates from the superolateral part of the deep femoral artery (DFA) and trifurcates into the ascending, transverse and descending branches constantly; type $\mathrm{Ib}$, the LFCA originates variably from the femoral artery (FA) but trifurcates into the ascending, transverse and descending branches constantly; type II, two trunks of the LFCA ascending, transverse and descending branches originate from the DFA or the FA; type III, the LFCA ascending, transverse and descending branches originate from the DFA or the FA, respectively; and type IV, one of the three LFCA branches is absent, most commonly the transverse branch (Table I).

\section{Results}

Origin pattern, trifurcation and perforating branches of LFCAs. As shown in Table I, the LFCAs of the bilateral lower limbs were well visualized by 3D-CE-MRA in all 68 patients. The anatomical pattern of the LFCA was type Ia in 80 cases $(58.8 \%)$, type Ib in 21 cases (15.4\%), type II in 20 cases (14.7\%), type III in 3 cases (2.2\%) and type IV in 12 cases $(8.8 \%)$. Tertiary vascular trees (primary, main trunk of LFCA; secondary, ascending, transverse and descending branches; tertiary, muscular perforating vessels) were identified in 94 limbs (69.1\%). The intermuscular orientation of the perforating vessels was also clearly shown with MRA. The length of the descending branch vascular pedicle (the linear distance from the origin of the branch to the distal end) ranged from 41 to $323 \mathrm{~mm}$. As shown in Fig. 1A and B, the left LFCA (type Ia) originated from the left DFA and subsequently trifurcated into the ascending, transverse and descending branches. As shown in Fig. 2A and B, the right LFCA (type Ib) originated variably from the right DFA and subsequently trifurcated into the ascending, transverse and descending branches. As shown in Fig. 3A and B, the descending branches of the bilateral femoral circumflex arteries converged with the ascending branch of the bilateral superior genicular arteries. Type Ib LFCA, i.e. an LFCA originating variably from the FA, was identified in 21 cases. Among these cases, TS-MIP reconstruction of the opening portion of femoral artery revealed the 
Table I. Anatomical classification of the LFCA $(n=136)$.

\begin{tabular}{|c|c|c|}
\hline Type & $\mathrm{n}(\%)$ & Definition \\
\hline I & $101(74.3)$ & \\
\hline Ia & $80(58.8)$ & $\begin{array}{l}\text { The LFCA originated from the superolateral part of the DFA and trifurcated into the ascending, } \\
\text { transverse and descending branches constantly. }\end{array}$ \\
\hline $\mathrm{Ib}$ & $21(15.4)$ & $\begin{array}{l}\text { The LFCA originated variably from the FA but trifurcated into the ascending, transverse and } \\
\text { descending branches constantly. }\end{array}$ \\
\hline II & $20(14.7)$ & $\begin{array}{l}\text { Two trunks of the LFCA ascending, transverse and descending branches originated from the } \\
\text { DFA or the FA. }\end{array}$ \\
\hline III & $3(2.2)$ & $\begin{array}{l}\text { The LFCA ascending, transverse and descending branches originated from the DFA or the FA, } \\
\text { respectively. }\end{array}$ \\
\hline IV & $12(8.8)$ & One of the three LFCA branches was absent, most commonly the transverse branch. \\
\hline
\end{tabular}

LFCA, lateral femoral circumflex artery; DFA, deep femoral artery; FA, femoral artery.
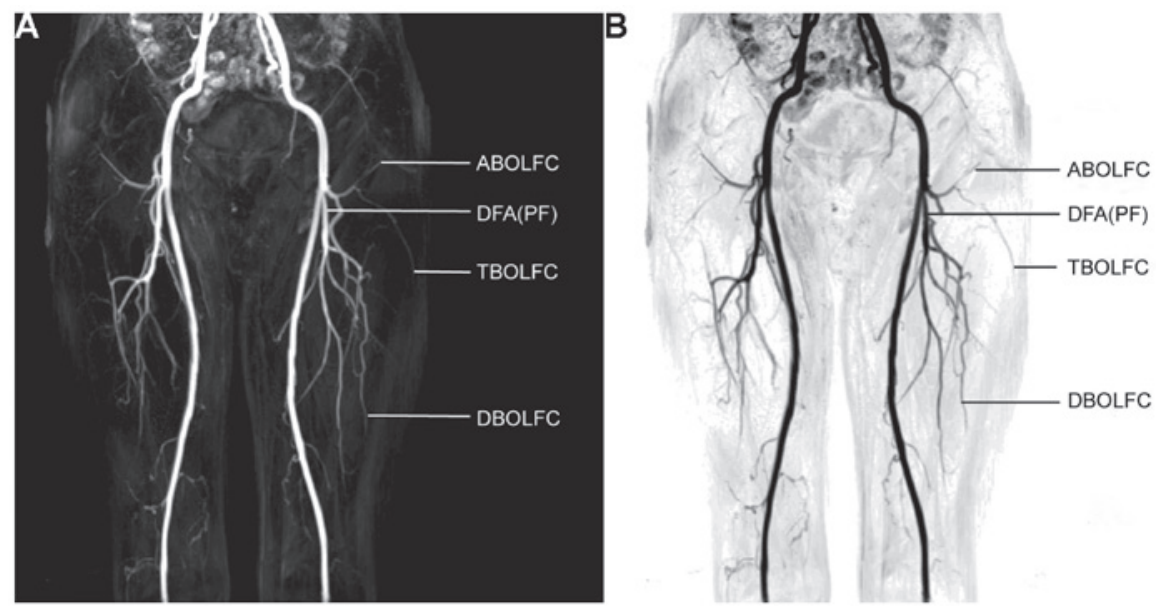

Figure 1. Type Ia LFCA. (A) Three dimensional-contrast-enhanced magnetic resonance angiography reconstructed maximum intensity projection and (B) digital subtraction angiography images showed that the LFCA originated from the DFA and subsequently trifurcated into the ascending, transverse and descending branches. LFCA, lateral femoral circumflex artery; DFA (PF), deep femoral artery (profunda femoris); ABOLFC, ascending branch of the LFCA; TBOLFC, transverse branch of the LFCA; DBOLFC, descending branch of the LFCA.

specific origin of the LFCA to be from the anterolateral part of the FA in 10 cases and from the posterolateral part of the FA in 11 cases, and another from the inferior part of the DFA in 19 cases.

LFCA perforating branches and flap outline. All three-tier LFCA branches were well visualized in subtractive 3D-MRA. Thick-slab MIP revealed the distribution and spatial pattern of the vessels, and TS-MIP ignored unrelated data to better visualize the target anatomy, namely the LFCA and its secondary and tertiary branches (Fig. 4A and B). The descending branch of the right LFCA was found to trifurcate into three parallel perforating arteries (Fig. 5A and B). The space between the terminal perforating arteries and the skin was clearly visualized to help in the decision of the surgical approach and the evaluation of flap outlining. The proposed flap was therefore primarily supplied by the LFCA descending branch. By contrast, the left LFCA descending branch extended into a single artery, without visible perforating arteries (Fig. 5C and D). The LFCA transverse branch was shown to be proximal to the skin, and its caliber could be precisely evaluated using MRA. For each patient, flap size was adjusted based on the surface area of limb cutaneous and subcutaneous tissue defects. According to the preoperative MRA results, the flaps were designed to encompass the center of the blood vessel (perforating artery pedicle), and typically $5-8 \mathrm{~cm}$ diameter flaps were appropriate.

Case reports. FALTF transfer was performed in 4 patients to cover the skin and soft tissue defects in the feet/pedal dorsum $(n=3)$ or hand dorsum $(n=1)$ (Fig. 6A-D). The preoperative evaluation results of the limb soft tissue defects are shown in Table II. CE-MRA with TS-MIP was used to outline the flap based on the distance of the perforating vessels to the skin, the calibers of the perforating vessels and the flap blood supply. Preoperative CE-MRA assessment offered an accurate localization of the perforating artery pedicles, which originated from the LFCA descending branch in 3 patients and from the LFCA transverse branch in 1 patient. The intraoperative measurements were identical to the preoperative 

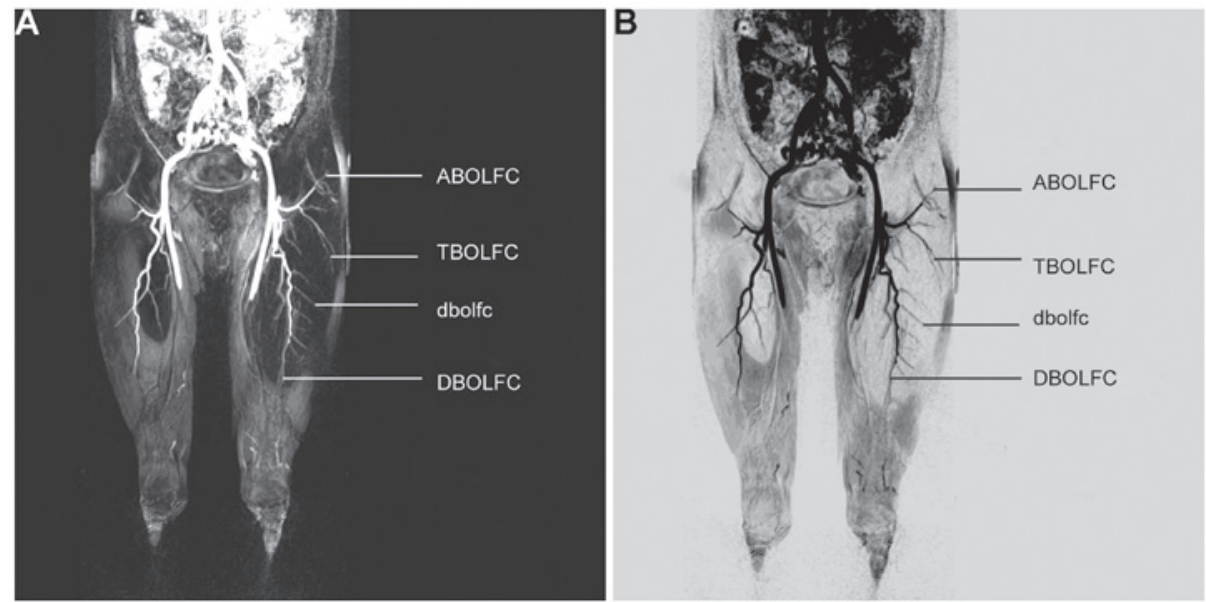

Figure 2. Type Ib LFCA. (A) Three dimensional-contrast-enhanced magnetic resonance angiography reconstructed maximum intensity projection and (B) digital subtraction angiography images showed that the LFCA originated variably from the femoral artery and subsequently trifurcated into the ascending, transverse and descending branches. LFCA, lateral femoral circumflex artery; ABOLFC, ascending branch of the LFCA; TBOLFC, transverse branch of the LFCA; DBOLFC, descending branch of the LFCA; dblofc, perforating branch of the LFCA.

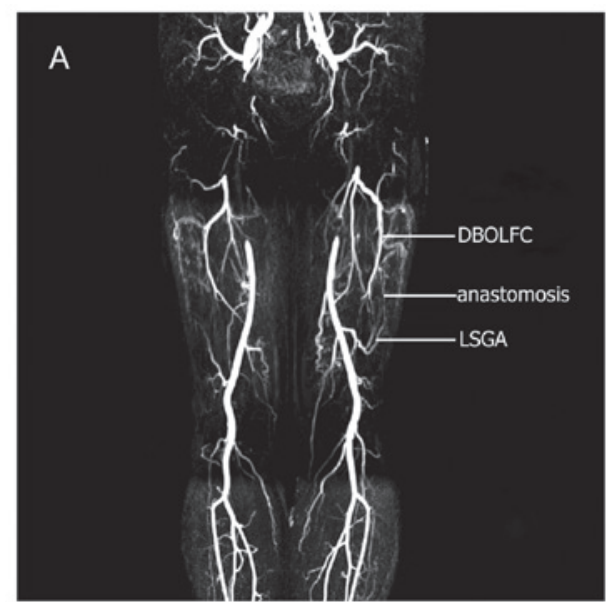

B

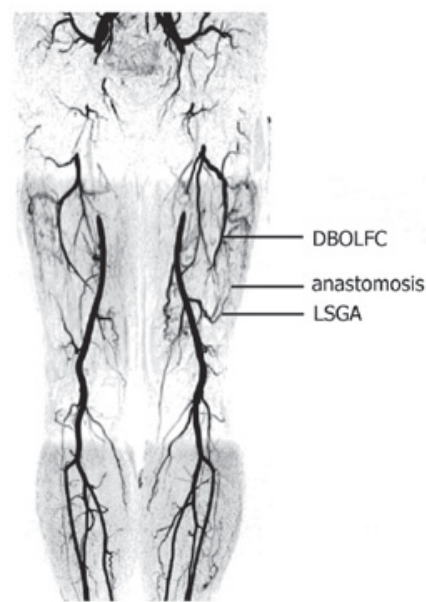

Figure 3. (A) Contrast-enhanced magnetic resonance angiography two-dimensional reconstructed thin-slab maximum intensity projection and (B) digital subtraction angiography images showing the bilateral superior genicular artery networks. DBOLFC, descending branch of the lateral femoral circumflex artery; LSGA, lateral superior geniculate artery.
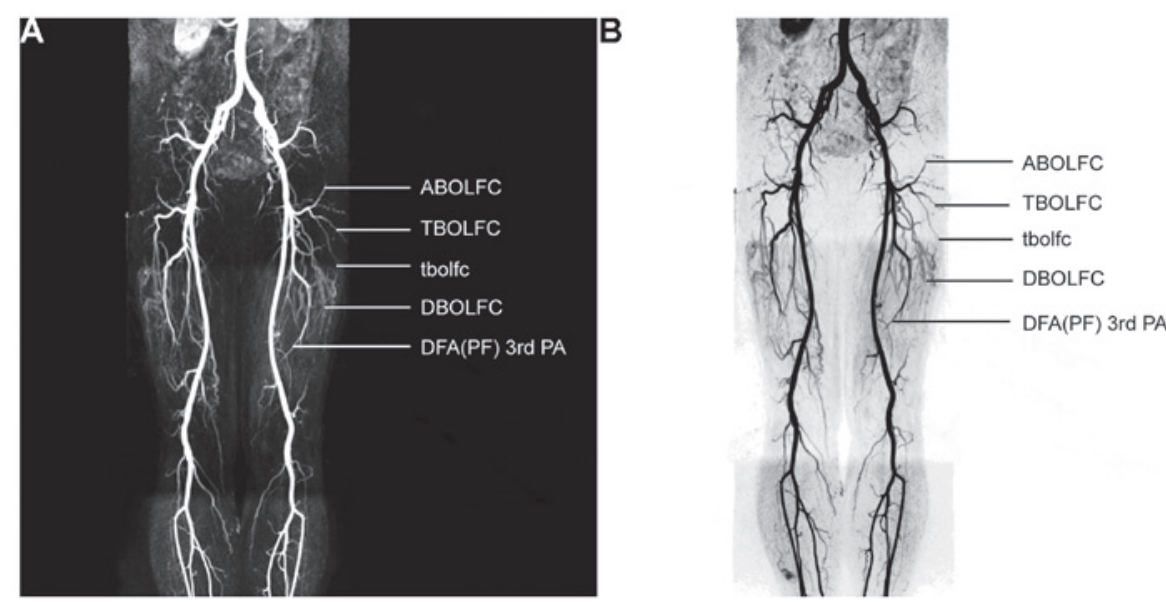

Figure 4. (A) Contrast-enhanced magnetic resonance angiography three-dimensional reconstructed maximum intensity projection and (B) digital subtraction angiography showed that the LFCA originated from the anterolateral part of the DFA. The pathways of the descending branch, the DFA and the superior genicular artery were well visualized. The perforating arteries arose from the transverse branch, and the space between the terminal perforating artery and the skin was also well visualized. LFCA, lateral femoral circumflex artery; ABOLFC, ascending branch of the LFCA; TBOLFC, transverse branch of the LFCA; tblofc, perforating branch of the LFCA; DBOLFC, descending branch of the LFCA; DFA (PF) 3rd PA, the third peripheral branch of the deep femoral artery (profunda femoris). 
MRA results in terms of the origin, localization, caliber and distribution pattern of the perforating vessels. The vascular caliber was adequate for flap transfer, and the flap size was appropriate for covering the wound. All patients underwent an uneventful flap transfer, without missing any perforating vessels or requiring additional flap outlining. All flaps were well perfused and survived the transfer. All wounds healed by primary intention, and no surgical site infection or wound dehiscence occurred.

\section{Discussion}

Normally, the LFCA trifurcates into three branches: Ascending, transverse and descending. The ascending branch runs in the tensor fasciae latae muscle and the anterolateral part of the iliac crest; the transverse branch advances into the anterolateral part of the greater trochanter; and the descending branch supplies the lateral femoral muscles and the ALT skin. The soft tissues nourished by the three LFCA branches are frequently harvested as flaps for repairing large-area soft tissue defects $(3,4)$. The use of a FALTF with a high-level cutaneous perforating artery pedicle has been reported in previous studies $(8,12,13)$. This flap preserves the subcutaneous adipose tissue for covering soft tissue defects; however, the revision of subcutaneous adipose tissue converts the flap into a thin flap with a dermal vascular network. This type of flap has been widely used, therefore, in the repair and reconstruction of the hand, head and face $(8,14,15)$.

Successful flap transfer requires an accurate preoperative assessment of the perforating vessels and their convergence. Thus, preoperative outlining of FALTF depends on the anatomical investigation of the LFCA perforating arteries. Currently, Doppler ultrasonography using the ABC method and CE Doppler ultrasound angiography are typically used by orthoplastic surgeons for preoperative body surface marking (14). These methods, however, only lead to an empirical decision of access approaches, and the proposed flaps are temporarily outlined in accordance with the intraoperative finding of vascular localization. CTA is used as one of the mainstay techniques for the preoperative assessment of ALT flaps, and has shown favorable results in current practice (15). In the present study, 3D-CE-MRA offered a direct, accurate visualization of the vascular anatomy without any clinically significant adverse effects, and consequently enabled surgeons to better outline the intended flap based on the preoperative anatomical evaluation.

The present results showed that the LFCA arose from the DFA in $82.3 \%$ of patients and from the FA in $15.4 \%$ of patients; however, our results were marginally different from those reported by Uzel et al (11) in 2008, who found that the LFCA was branched from the DFA in $77.3 \%$ of cases and from the FA in $19.1 \%$ of cases. Since Luo (1) and Chen (2) initially described the classification of LFCA branching patterns, a number of modified classifications have been reported, such as those of Kimata et al (16) and Shieh et al (12). In the present study, we proposed another modified classification in line with the LFCA branching patterns on MRA: Type Ia, the LFCA originates from the superolateral part of the deep FA and trifurcates into the ascending, transverse and descending branches constantly; type $\mathrm{Ib}$, the LFCA originates variably 

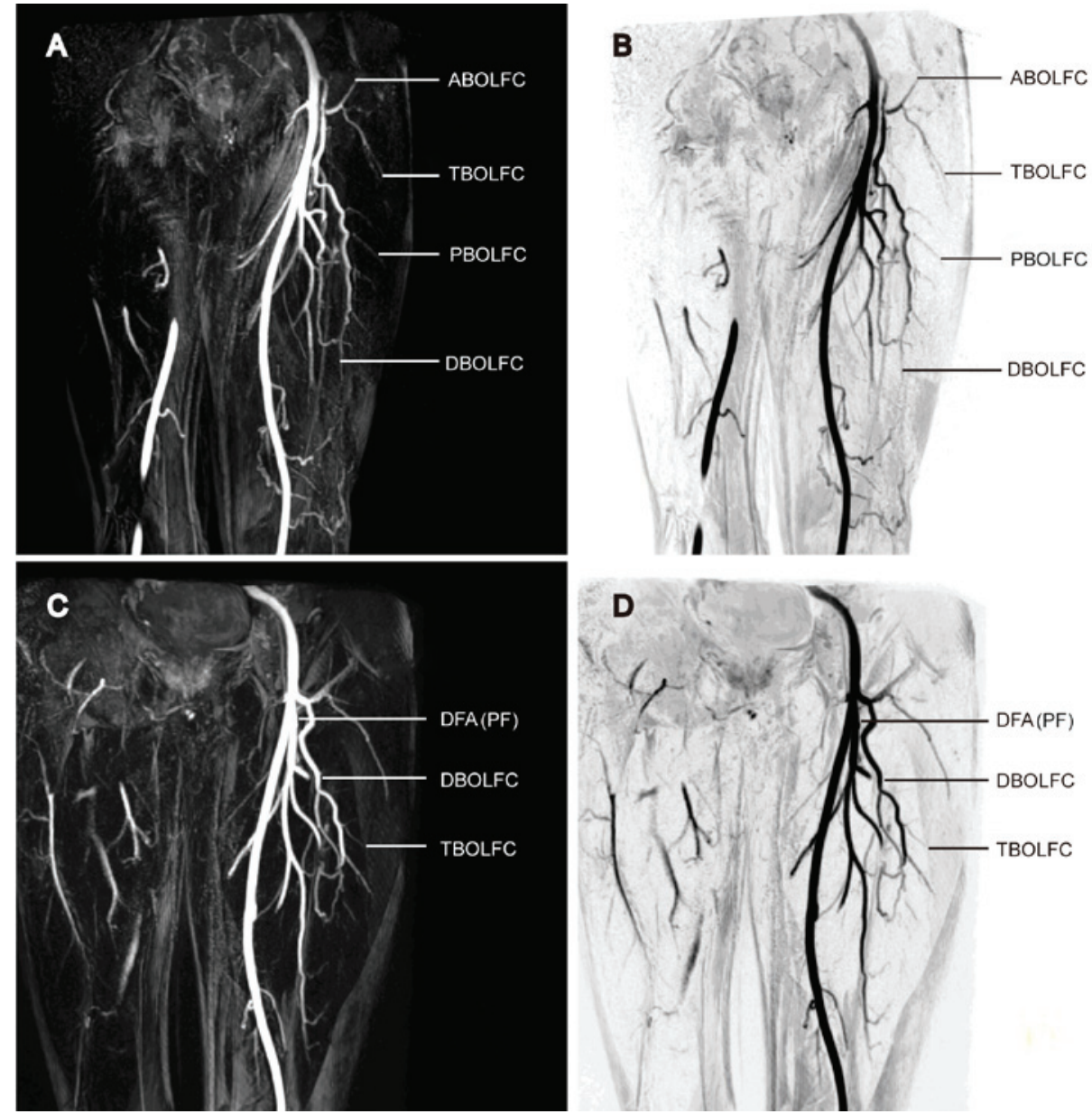

Figure 5. (A and C) Contrast-enhanced magnetic resonance angiography two-dimensional reconstructed thin-slab maximum intensity projection and (B and D) digital subtraction angiography images showing the LFCA and its branches. LFCA, lateral femoral circumflex artery; ABOLFC, ascending branch of the LFCA; TBOLFC, transverse branch of the LFCA; PBOLFC, perforating arteries of the LFCA; DBOLFC, descending branch of the LFCA; DFA (PF), deep femoral artery (profunda femoris).

from the FA but trifurcates into the ascending, transverse and descending branches constantly; type II, two trunks of the LFCA ascending, transverse and descending branches originate from the DFA or the FA; type III, the LFCA ascending, transverse and descending branches originate from the DFA or the FA, respectively; and type IV, the LFCA originates from the DFA and bifurcates into the ascending and descending branches only, with the transverse branch missing. In terms of hemodynamics, this modified classification describes the LFCA origin more accurately than the description by Uzel et al (11) in 2008. This modified classification also enables surgeons to precisely evaluate the dynamics of the major LFCA branches for outlining a variety of skin flaps or composite tissue flaps $(6,17)$.

It has been well established that the preoperative outlining for perforating artery flaps follows four primary principles: 'Point', 'line', 'plane' and 'arch'. Perforating vessels are ideally localized prior to surgery, with Doppler ultrasonography frequently used for preoperative flap vascular investigation. This technique is minimally invasive, easy to use and less costly; however, it is unable to determine the vascular origin and is subject to a high false-positive rate. Furthermore, Doppler ultrasonography has low sensitivity and accuracy, and cannot offer visual images for intraoperative use, due to the absence of hologram and 3D anatomical reconstructions, even for experienced operators $(18,19)$. Multiple intrinsic disadvantages are also associated with DSA, such as high cost, invasiveness, risk of iatrogenic injuries and low reproducibility. In addition, DSA imaging cannot visualize muscles, nerves, fat and other soft tissues, resulting in an incomplete visualization of soft tissues. DSA does, however, allow a clearer visualization of the perforating vessels than CTA or MRA and, therefore, remains the gold standard technique for imaging the vascular 3D anatomy. Multi-detector CTA can reconstruct the 3D anatomy of vessels located in the flap and surgical field but requires delicate equipment; this technique is also subject to high costs and a risk of potential radiation injury (20-22).

Newly emerging 3D-CE-MRA has been incorporated in vascular interventions (23), although it is rarely reported in the evaluation of LFCA perforating vessels. This medical imaging modality is easy to use and presents a high success rate (24). Furthermore, TS-MIP allows an objective, visual assessment of vascular origin, perforating vessel distribution and vascular pedicle measurement. This technique offers a dynamic patterning of intermuscular perforating vessels and neighboring vascular networks. As it is a minimally invasive, visual and accurate imaging technique, 3D-CE-MRA is considered 

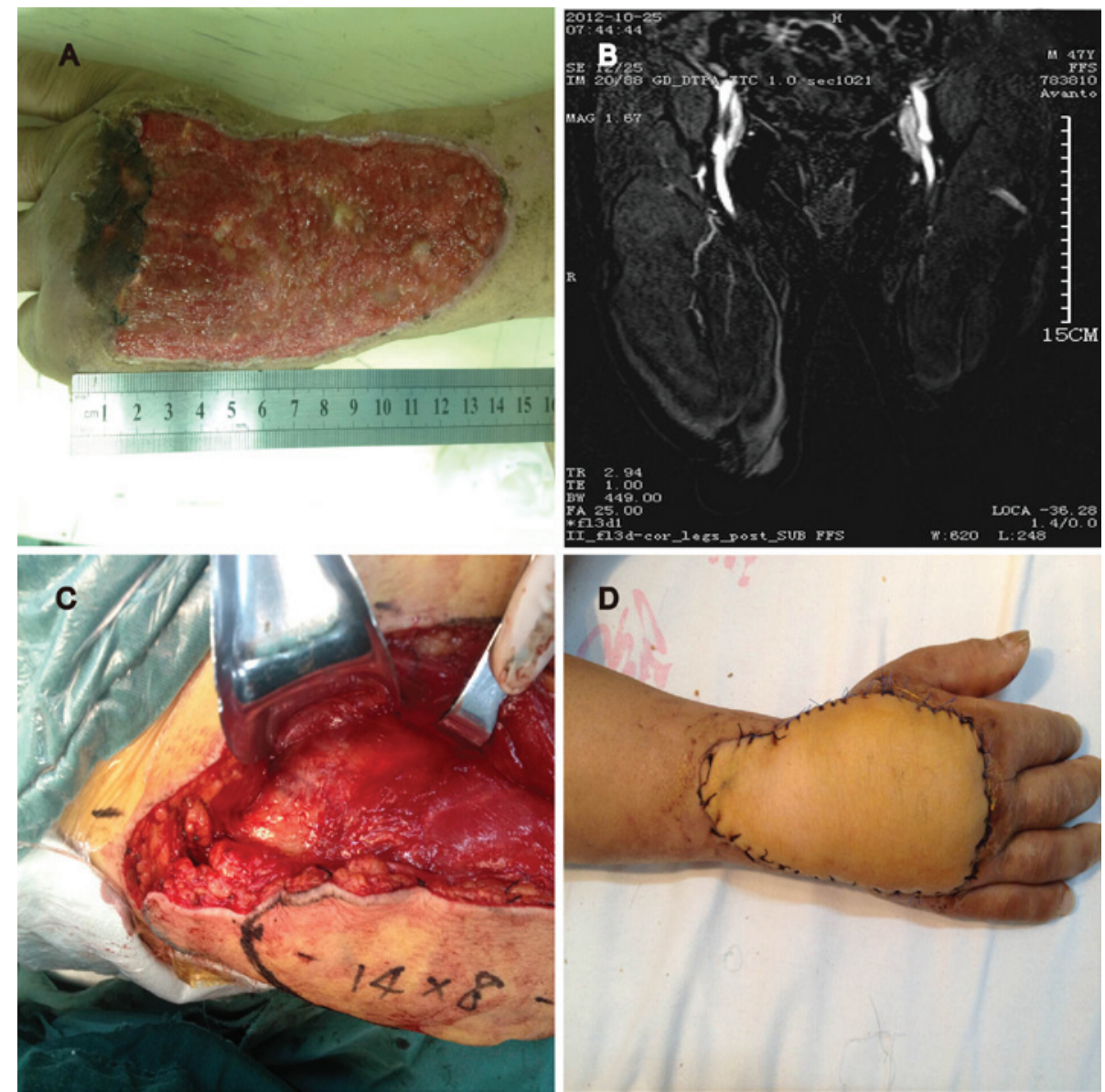

Figure 6. A 46-year-old male suffered from right hand crushing injury, which resulted in dorsum skin and tendon defects with complicating bone exposure. Multiple sessions of debridement and wound dressing were given, and healthy granulation tissues emerged in the wound 14 days after the injury. (A) Physical appearance of the skin defect at a size of $13 \times 7 \mathrm{~cm}$. (B) 3D-CE-MRA with TS-MIP evaluation of the LFCA trifurcation and perforating branches; TS-MIP showed the path of various branches and perforators of the LFCA within the intermuscular space. (C) Outlining a 14x8 $\mathrm{cm}$ free anterolateral thigh flap; the intraoperative finding was consistent with the 3D-CE-MRA result. (D) Physical appearance of the covered hand dorsum wound at 2 weeks from the day of flap transfer, showing a surviving flap and a well-healed wound. 3D-CE-MRA, three-dimensional contrast-enhanced magnetic resonance angiography; TS-MIP, thin-slab maximum intensity projection; LFCA, lateral femoral circumflex artery.

to be an effective preoperative assessment tool. This technique has numerous advantages (25). First, CE-MRA allows simultaneous flap outlining at multiple sites. Secondly, CE-MRA offers a 3D, full-scale, multi-angle reconstruction of vessels, which is particularly useful in cases requiring additional flap outlining due to an inability to identify an appropriate perforating artery. Thirdly, CE-MRA can be used for the postoperative evaluation of flaps and adjoining soft tissues in case of poor perfusion. These advantages were validated by the present results from 4 patients undergoing 3D-CE-MRA for FALTF outlining.

The techniques of Test Bolus and Care Bolus $(26,27)$ may be used in 3D-CE-MRA. The first method measures the time from zero to contrast peak concentration, followed by MRA imaging, resulting in high quality angiography but requiring a relatively experienced operator. The second method uses real-time tracing and trigger acquisition and is easy to use in general practice, although it also requires an experienced operator. In the present study, the latter method, i.e. the real-time tracing and trigger acquisition method, was used. The dose of contrast media was determined to be $\sim 30 \mathrm{ml}$ in the majority of patients due to the following two reasons: Double-dose contrast media offers a better-visualized vasculature, and this dose is not likely to require any additional media for calf MRA. A higher bolus injection rate results in a more legible contrast-enhanced artery, as well as perforating vessel convenience and lateral circulation; however, the peak time is relatively short in case of a high injection rate, which builds up challenges in MRA scanning and requires the use of a magnetic resonance scanner with a high temporal resolution. We therefore proposed an injection rate of $\sim 3.0 \mathrm{ml} / \mathrm{sec}(2.5-4.0 \mathrm{ml} / \mathrm{sec}$ in the patients), which achieved a favorable angiography peak time and also avoided potential local injuries from contwrast medium leakage. For angiography from the common iliac artery to the superior genicular artery, multiple serial scanning should be repeated, if possible, to visualize arteries and veins simultaneously. The results from the present study validated the excellent outcome of this approach, as the LFCA was well visualized in all patients, in terms of origin, pathway and distribution.

The MIP technique is normally used for post-angiography processes, and allows the visualization of the target anatomy, either positively or negatively. Images derived from the Care Bolus approach are similar to those from DSA, and allow the review against the DSA finding, which is particularly suitable for reviewers with DSA experience $(28,29)$. The incorporation 
of TS-MIP excludes the interference from a large number of unrelated structures and preserves legible, realistic images; therefore, this technique only visualizes the target anatomy and better characterizes the flap vascular architecture and distribution. In the present study, MRA with TS-MIP visualized all three-tier LFCA branches in the majority of donor sites and aided surgeons to outline the flap prior to surgery.

There were a number of limitations in this study. First, only the static 3D anatomy of the LFCA was demonstrated, as well as its major branches and perforating vessels, rather than the dermal vascular network. Secondly, the determination of the flap size was neither prospective nor accurate. Finally, the sample size was relatively small. Our ongoing study aims to quantitatively evaluate the donor site blood supply and flap size using MRA with 3D perfusion imaging.

In conclusion, 3D-CE-MRA is a clinically effective investigational modality in evaluating the 3D anatomy of LFCA for FALTF transfer; however, the effectiveness and safety of this technique require validation in further large-scale, prospective, randomized, controlled studies.

\section{References}

1. Luo LS: A new free skin flap-anterolateral femoral flap - its anatomy and clinical application. Zhonghua Zheng Xing Shao Shang Wai Ke Za Zhi 1: 50-52, 1985 (In Chinese).

2. Chen LF: The application of a free anterolateral femoral cutaneous flap for the repair of chronic ulcers of foot and ankle. Zhonghua Zheng Xing Shao Shang Wai Ke Za Zhi 3: 118-119, 157, 1987 (In Chinese).

3. Zhang B, Li DZ, Xu ZG and Tang PZ: Free anterolateral thigh flap for reconstruction of head and neck defects. Zhonghua Er Bi Yan Hou Tou Jing Wai Ke Za Zhi 41: 447-450, 2006 (In Chinese).

4. Park CW and Miles BA: The expanding role of the anterolateral thigh free flap in head and neck reconstruction. Curr Opin Otolaryngol Head Neck Surg 19: 263-268, 2011.

5. Sharabi SE, Hatef DA, Koshy JC, et al: Is primary thinning of the anterolateral thigh flap recommended? Ann Plast Surg 65: $555-559,2010$.

6. Smit JM, Klein S and Werker PM: An overview of methods for vascular mapping in the planning of free flaps. J Plast Reconstr Aesthet Surg 63: e674-e682, 2010.

7. Garvey PB, Selber JC, Madewell JE, et al: A prospective study of preoperative computed tomographic angiography for head and neck reconstruction with anterolateral thigh flaps. Plast Reconstr Surg 127: 1505-1514, 2011.

8. Liu SC, Chiu WK, Chen SY, et al: Comparison of surgical result of anterolateral thigh flap in reconstruction of through-and-through cheek defect with/without CT angiography guidance. J Craniomaxillofac Surg 39: 633-638, 2011.

9. Hnatiuk B, Emery DJ and Wilman AH: Effects of doubling and tripling the spatial resolution in standard 3D contrast-enhanced magnetic resonance angiography of carotid artery disease. J Magn Reson Imaging 27: 71-77, 2008.

10. Lakhiani C, Lee MR and Saint-Cyr M: Vascular anatomy of the anterolateral thigh flap: A systematic review. Plast Reconstr Surg 130: 1254-1268, 2012.

11. Uzel M, Tanyeli E and Yildirim M: An anatomical study of the origins of the lateral circumflex femoral artery in the Turkish population. Folia Morphol (Warsz) 67: 226-230, 2008.

12. Shieh SJ, Chiu HY, Yu JC, et al: Free anterolateral thigh flap for reconstruction of head and neck defects following cancer ablation. Plast Reconstr Surg 105: 2349-2357, 2000.
13. Rastogi S, Patwardhan B, Gulati A and Thayath $\mathrm{MN}$ : Anterolateral thigh free flap for the reconstruction of through and through defect of cheek following cancer ablation. Indian J Dent Res 23: 275-278, 2012.

14. Donnelly R, Hinwood D and London NJ: ABC of arterial and venous disease. Non-invasive methods of arterial and venous assessment. BMJ 320: 698-701, 2000.

15. Chen SY, Lin WC, Deng SC, Chang SC, Fu JP, Dai NT, Chen SL, Chen TM and Chen SG: Assessment of the perforators of anterolateral thigh flaps using 64-section multidetector computed tomographic angiography in head and neck cancer reconstruction. Eur J Surg Oncol 36: 1004-1011, 2010.

16. Kimata Y, Uchiyama K, Ebihara S, et al: Anatomic variations and technical problems of the anterolateral thigh flap: A report of 74 cases. Plast Reconstr Surg 102: 1517-1523, 1998.

17. Xu D, Zhang S, Tang M and Ouyang J: Development and current status of perforator flaps. Zhongguo Xiu Fu Chong Jian Wai Ke Za Zhi 25: 1025-1029, 2011 (In Chinese).

18. Masia J, Larrañaga J, Clavero JA, et al: The value of the multidetector row computed tomography for the preoperative planning of deep inferior epigastric artery perforator flap: Our experience in 162 cases. Ann Plast Surg 60: 29-36, 2008.

19. Rozen WM, Garcia-Tutor E, Alonso-Burgos A, et al: Planning and optimising DIEP flaps with virtual surgery: The Navarra experience. J Plast Reconstr Aesthet Surg 63: 289-297, 2010.

20. Jia Y, Liu W, Zeng A, et al: Clinical application of multidetector row CT angiography for preoperative evaluation of nourished vessels of flaps. Zhonghua Zheng Xing Wai Ke Za Zhi 24: 275-278, 2008 (In Chinese).

21. Ribuffo D, Atzeni M, Saba L, et al: Angio computed tomography preoperative evaluation for anterolateral thigh flap harvesting. Ann Plast Surg 62: 368-371, 2009.

22. Saint-Cyr M, Schaverien M, Wong C, et al: The extended anterolateral thigh flap: Anatomical basis and clinical experience. Plast Reconstr Surg 123: 1245-1255, 2009.

23. Lin J, Li D and Yan F: High-resolution 3D contrast-enhanced MRA with parallel imaging techniques before endovascular interventional treatment of arterial stenosis. Vasc Med 14: 305-311, 2009

24. Tizon X, Lin Q, Hansen T, Borgefors G, Johansson L, Ahlström H and Frimmel H: Identification of the main arterial branches by whole-body contrast-enhanced MRA in elderly subjects using limited user interaction and fast marching. J Magn Reson Imaging 25: 806-814, 2007.

25. Schaverien MV, Ludman CN, Neil-Dwyer J and McCulley SJ: Contrast-enhanced magnetic resonance angiography for preoperative imaging of deep inferior epigastric artery perforator flaps: Advantages and disadvantages compared with computed tomography angiography: A United Kingdom perspective. Ann Plast Surg 67: 671-674, 2011.

26. Kramer H, Zenge M, Schmitt P, et al: Peripheral magnetic resonance angiography (MRA) with continuous table movement at $3.0 \mathrm{~T}$ : Initial experience compared with step-by-step MRA. Invest Radiol 43: 627-634, 2008.

27. Butz B, Dorenbeck U, Borisch I, et al: High-resolution contrast-enhanced magnetic resonance angiography of the carotid arteries using fluoroscopic monitoring of contrast arrival: Diagnostic accuracy and interobserver variability. Acta Radiol 45: 164-170, 2004.

28. Neil-Dwyer JG, Ludman CN, Schaverien M, et al: Magnetic resonance angiography in preoperative planning of deep inferior epigastric artery perforator flaps. J Plast Reconstr Aesthet Surg 62: 1661-1665, 2009.

29. Schaverien MV, Ludman CN, Neil-Dwyer J and McCulley SJ: Contrast-enhanced magnetic resonance angiography for preoperative imaging of deep inferior epigastric artery perforator flaps: advantages and disadvantages compared with computed tomography angiography: A United Kingdom perspective. Ann Plast Surg 67: 671-674, 2011. 